\title{
Accurate Quasinormal Modes of the Five-Dimensional Schwarzschild-Tangherlini Black Holes
}

\author{
Jerzy Matyjasek ${ }^{*}$ \\ Institute of Physics, Maria Curie-Sktodowska University \\ pl. Marii Curie-Sktodowskiej 1, 20-031 Lublin, Poland
}

\begin{abstract}
The objective of this paper is to construct the accurate (say, to 11 decimal places) frequencies of the quasinormal modes of the 5-dimensional Schwarzschild-Tangherlini black hole using three major techniques: the Hill determinant method, the continued fractions method and the WKB-Padé method and to discuss the limitations of each. It is shown that for the massless scalar, gravitational tensor, gravitational vector and electromagnetic vector perturbations considered in this paper, the Hill determinant method and the method of continued fractions (both with the convergence acceleration) always give identical results, whereas the WKB-Padé method gives the results that are amazingly accurate in most cases. Notable exception are the gravitational vector perturbations $(j=2$ and $\ell=2)$, for which the WKB-Padé approach apparently does not work. Here we have interesting situation in which the WKB-based methods (WKB-Padé and WKB-Borel-Le Roy) give the complex frequency that differs from the from the result obtained within the framework of the continued fraction method and the Hill determinant method. For the fundamental mode, deviation of the real part of frequency from the exact value is $0.5 \%$ whereas the deviation of the imaginary part is $2.7 \%$. For $\ell \geq 3$ the accuracy of the WKB results is similar again to the accuracy obtained for other perturbations. The case of the gravitational scalar perturbations is briefly discussed.
\end{abstract}

\section{INTRODUCTION}

The quasinormal modes of the static and spherically symmetric black holes are the solutions of the ordinary second-order Schrödinger-like differential equation

$$
\frac{d^{2}}{d x^{2}} \psi+\left(\omega^{2}-V[r(x)]\right) \psi=0
$$

where $V[r(x)]$ is the potential, $x$ is the tortoise coordinate and $\psi=\psi[r(x)]$ describes the radial perturbations, satisfying the purely outgoing boundary conditions at infinity and purely ingoing at the horizon. We assume that the potential is constant as $|x| \rightarrow \infty$ (the limits may be different) and has a maximum at some point $x_{0}$. The complex frequencies of the quasinormal modes, $\omega$, the

*Electronic address: jurek@kft.umcs.lublin.pl, jirinek@gmail.com 
real part of which gives the frequency of the oscillation and the imaginary part describes damping of the signal, are labeled by the spin weight, $j$, the multipole number, $\ell$, and the overtone number $n$. Since their discovery, the quasinormal modes have been the subject of intense research and debate. Although currently we have a good understanding of their nature and the reliable results at our disposal span from wormholes to black holes and from pulsars to analogue black holes, there are still more questions than answers. The interested reader can consult a few excellent review papers [1] 4]. (See also Ref. [5]).

A huge number of results that have been obtained so far can be classified with respect to the type and dimension of the black hole, the type of the perturbations, adapted methods and accuracy. The more specific classification may differentiate between long-lived and highly-damped modes, presence of the algebraically special solutions and the aims of the researcher. In this paper we shall concentrate on the static and spherically-symmetric black holes described by the Schwarzschild-Tangherlini line element

$$
d s^{2}=f(r) d t^{2}-f^{-1}(r) d r^{2}-r^{2} d \Omega_{D-2}^{2}
$$

with

$$
f(r)=1-r^{3-D},
$$

where $D$ is the dimension. Our aim is twofold. First, we calculate the highly accurate (accurate to, say, 11 decimal places) complex frequencies of the quasinormal modes of the five-dimensional Schwarzschild black holes for the massless scalar, gravitational tensor, gravitational vector and electromagnetic vector perturbations. In this regard it can be thought of as an extension of the important papers [6, 7]. The methods of choice are the WKB-Padé method, the Hill determinant method and the method of continued fractions. Second, we compare the methods and the accuracy of the results they give. We shall accept the result only if at least two methods agree to the assumed accuracy. All the complex frequencies presented in this paper do satisfy this requirement, and in most cases there is full agreement of all three approaches. Additionally, each result obtained with the Hill determinant method, has been, in fact, obtained in the course of the two independent calculations using the four-term and the three-term recurrences. Similarly, the method of continued fraction has been implemented in two different ways.

Only the last of the three methods is well-known and widely used. Indeed, since the publication of the Leaver's paper [8] in 1985, the continued fractions method (with smaller or greater modifications) have been successfully employed in numerous cases. Usually, it is described as robust, highly 
accurate, stable and reliable. On the other hand, the Hill determinant method [9] is less known. It is criticized for losing the accuracy of the higher-order overtones. However, such behavior is also typical for other methods. It should be emphasized that in the continued fractions method one can find asymptotic expression describing the tail (i.e., the remaining part of the infinite continued fraction) that certainly improves the quality of approximants. All this reduced the Hill determinant method to the inferior role of supplier of the initial values of $\omega$ for more mature methods. In this paper however, we shall propose an extension of the Hill determinant method, which, in our opinion, partially answers the criticism pronounced by some authors.

Finally, the WKB-Padé method [10-12] is a recent extension and modification of the IyerWill approach [13] (and its further generalizations [14]). It differs from the previously discussed methods in several aspects, the most important of which is its 'black box' nature with the potential $V(x)$ treated as an input and the accurate quasinormal modes as the output. As in other WKBbased methods, the frequency of the quasinormal modee is (formally) given by a series of terms constructed, for a given $j$ and $l$, solely from the derivatives of the potential $V(x)$ at $x_{0}$. Since this series is divergent, one can try to use some standard techniques to obtain sensible results. For this purpose, it has been proposed to apply the Padé transform to the the truncated series. This suggests that the WKB-Padé method could be, in principle, applicable to a wide class of potentials. Indeed, it has been shown that in many cases this procedure, despite its simplicity, yields amazingly accurate results. For example, and it is really impressive, all three methods give (to 32 digits accuracy) exactly the same frequency for the lowest fundamental mode of the gravitational perturbations of the Schwarzschld black hole.

$$
\omega=0.74734336883608367158698400595410-0.17792463137787139656092185436905 i .
$$

Moreover, to 28 digits accuracy this result is confirmed by independent calculations employing the confluent Heun functions [15].

The literature on the quasinormal modes of the higher-dimensional Schwarzschild-Tangherlini black hole is quite rich. For problems not covered here the interested reader may consult, for example, Refs. [16-20] and the references cited therein. Specifically, we shall not discuss behavior of the quasinormal modes which can easily be inferred form low accuracy calculations. The ideal situation we have in mind is the following: Suppose that we have very accurate measurements of the quasinormal frequencies $\omega$ and our task is to choose between the black hole models. From the astrophysical point of view the most important ones are the long lived modes, such as considered here. Our second goal, beyond natural curiosity, is to compare accuracy and overall performance 
of various competing methods. Finally, it should be emphasized that in order to understand its pros and cons, every new method should be extensively tested against the existing accurate results. Consequently the role played by the highly accurate values of the quasinormal frequencies should not be underestimated.

The paper is organized as follows. In Sec. II we provide basic equations and introduce the calculational strategies. Specifically, in Sec. II A we discuss the Hill determinant method. To the author's knowledge it is a first attempt to improve quality of the method by using the series acceleration techniques in the construction of the complex frequencies of the quasinormal modes and a first attempt to employ the Hill determinant method for the perturbations of the higherdimensional black holes. We also show, that the Gauss elimination is not necessary ingredient for the calculations involving 4-term recurrence relations. (See also Ref. [21]). In Sec. II B]we introduce the continued fraction method with the series acceleration and in Sec. IIC we briefly discuss the WKB-Padé method. In Sec. IIIA we present the results of the calculation of the quasinormal frequencies of massless scalar and gravitational tensor perturbations. We follow the normalization used in Ref. [6]. Similarly, in Sec. IIIB we discuss our results for gravitational and electromagnetic vector perturbations. Finally, in Sec. IV we briefly discuss our preliminary calculations of the quasinormal frequencies of the gravitational scalar perturbations.

\section{THE QUASINORMAL FREQUENCIES OF 5-DIMENSIONAL SCHWARZSCHILD-TANGHERLINI BLACK HOLES}

The differential (master) equation describing the massless scalar, gravitational tensor, gravitational vector and electromagnetic vector perturbations of the $D$-dimensional SchwarzschildTangherlini black hole [22 25] $(D>4)$ can be written in a compact form:

$$
\frac{d^{2}}{d x^{2}} \psi+\left\{\omega^{2}-f(r)\left[\frac{l(l+D-3)}{r^{2}}+\frac{(D-2)(D-4)}{4 r^{2}}+\frac{\left(1-j^{2}\right)(D-2)^{2}}{4 r^{D-1}}\right]\right\} \psi
$$

where $j$ is given by

$$
j= \begin{cases}0, & \text { massless scalar and gravitational tensor perturbations. } \\ 2, & \text { gravitational vector perturbations } \\ \frac{2}{D-2}, & \text { electromagnetic vector perturbations. }\end{cases}
$$

On the other hand, the potential of the gravitational scalar perturbations is more complicated

$$
V=\frac{f(r) Q(r)}{4 r^{2}[2 m+d(d+1) \xi]^{2}},
$$


where

$$
\begin{aligned}
Q(r)= & d^{4}(d+1)^{2} \xi^{3}+d(d+1)\left[4\left(2 d^{2}-3 d+4\right) m+d(d-2)(d-4)(d+1)\right] \xi^{2} \\
& -12 d[(d-4) m+d(d+1)(d-2)] m \xi+16 m^{3}+4 d(d+2) m^{2}
\end{aligned}
$$

$d=D-2, m=l(l+d-1)-d, \xi=r^{3-D}$. It does not belong to the class of potentials described by Eq. (5), and as such it will not be considered here in any depth. Only a few preliminary results obtained within the framework of the WKB-Padé method will be briefly discussed at the end of this paper. This case certainly deserves a separate and more thorough study.

Following [6], we shall therefore confine ourselves to the perturbations characterized by $j=$ $0,2 / 3$ and 2 . Since one of the aims of this paper is to perform the stress tests of the three methods briefly discussed in Introduction one can also use some 'unphysical' values of $j$. For the evendimensional black hole the perturbation function $\psi(r)$ can be expanded as a power series

$$
\psi(r)=\left(\frac{r-1}{r}\right)^{-i \omega /(D-3)} e^{i \omega r} \sum_{n=0}^{\infty} a_{n}\left(\frac{r-1}{r}\right)^{n},
$$

whereas for odd $D$ it can be expanded as

$$
\psi(r)=\left(\frac{r-1}{r+1}\right)^{-i \omega /(D-3)} e^{i \omega r} \sum_{n=0}^{\infty} a_{n}\left(\frac{r-1}{r}\right)^{n} .
$$

Upon substituting the expansions (9) and (10) into the master equation (5) one obtains a $(2 D-5)$ term recurrence relation and $(2 D-6)$-term recurrence relation, respectively.

Now, let us confine to the five-dimensional case. Making use of 10 , after some algebra, one obtains a four-term recurrence relations

$$
\begin{aligned}
& 0=\alpha_{0} a_{1}+\beta_{0} a_{0} \\
& 0=\alpha_{1} a_{2}+\beta_{1} a_{1}+\gamma_{1} a_{0} \\
& 0=\alpha_{k} a_{k+1}+\beta_{k} a_{k}+\gamma_{k} a_{k-1}+\delta_{k} a_{k-2}
\end{aligned}
$$

where

$$
\begin{aligned}
\alpha_{k} & =-2(k+1)(k-2 \rho+1), \\
\beta_{k} & =5 k^{2}+k(5-16 \rho)+l(l+2)+16 \rho^{2}-8 \rho+\frac{9}{4}\left(1-j^{2}\right)+\frac{3}{4}, \\
\gamma_{k} & =-4 k^{2}+8 k \rho-\frac{9}{2}\left(1-j^{2}\right)+4, \\
\delta_{k} & =k^{2}-k+\frac{9}{4}\left(1-j^{2}\right)-2
\end{aligned}
$$

and $\rho=i \omega / 2$. It should be noted that our recurrence relations differ from these presented in Ref. [6] and can easily be constructed or checked with the aid of any computer algebra system. 


\section{A. The Hill determinant method}

Inspection of the recurrence relations (11) shows that they define a sparse banded matrix $\mathcal{H}$ of the width $w=4$

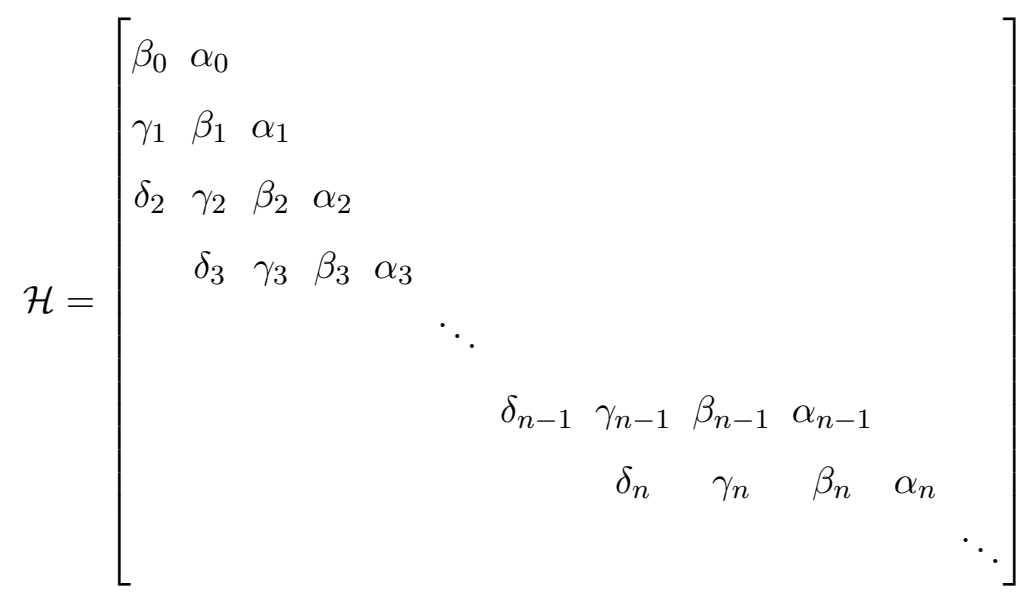

The condition that a nontrivial solution of the recurrence exists is given by the equation

$$
\operatorname{det} \mathcal{H}=0 \text {. }
$$

Now, let $h_{k}$ denotes determinant of $(k+1) \times(k+1)$ matrix constructed from the infinite matrix $\mathcal{H}$. Calculations of the determinants of such matrices using standard techniques may be impractical. The special form of $\mathcal{H}$ suggests another approach. Indeed, the Laplace expansion along $n$-th row gives simple recurrence formula

$$
h_{n}=\beta_{n} h_{n-1}-\gamma_{n} \alpha_{n-1} h_{n-2}+\delta_{n} \alpha_{n-1} \alpha_{n-2} h_{n-3} .
$$

Although Eq. (16) alone is sufficient for determination of the quasinormal modes, having in mind other applications, we transform the four-term recurrence relations to the three-term ones. It can easily be done with the aid of the Gauss elimination method. Standard manipulations give:

$$
\begin{gathered}
\alpha_{0}^{\prime} a_{1}+\beta_{0}^{\prime} a_{0}=0, \\
\alpha_{k}^{\prime} a_{k+1}+\beta_{k}^{\prime} a_{k}+\gamma_{k}^{\prime} a_{k-1}=0,
\end{gathered}
$$

where the primed coefficients are given by

$$
\begin{gathered}
\alpha_{k}^{\prime}=\alpha_{k}, \\
\beta_{k}^{\prime}=\beta_{k}, \\
\beta_{k}^{\prime}=\beta_{k}-\frac{\delta_{k}}{\gamma_{k-1}^{\prime}} \alpha_{k-1}^{\prime},
\end{gathered}
$$




$$
\begin{aligned}
\gamma_{k}^{\prime} & =\gamma_{k}, \\
\gamma_{k}^{\prime} & =\gamma_{k}-\frac{\delta_{k}}{\gamma_{k-1}^{\prime}} \beta_{k-1}^{\prime} .
\end{aligned}
$$

The thus obtained three-term recurrence defines a tridiagonal matrix $\mathcal{H}^{\prime}$ (a sparse banded matrix of width 3 )

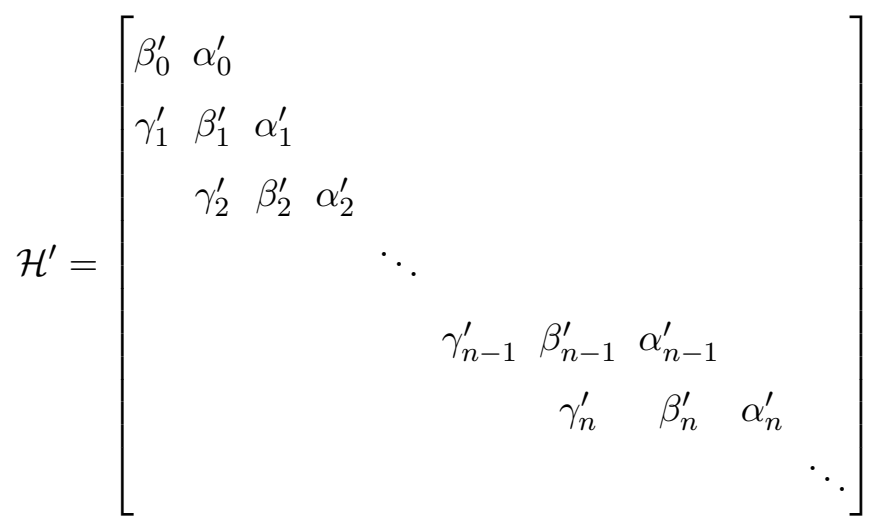

Denoting the determinant of $(k+1) \times(k+1)$ matrix by $h_{k}^{\prime}$, one has the following simple relation

$$
h_{n}^{\prime}=\beta_{n}^{\prime} h_{n-1}^{\prime}-\gamma_{n}^{\prime} \alpha_{n-1}^{\prime} h_{n-2}^{\prime},
$$

which (formally) follows from the previous one by putting $\delta_{k}=0$.

The general idea of the calculations is as quite simple: We truncate the series expansion at some $n$, and calculate the determinant, which is a polynomial $(w=4)$ or a rational function $(w=3)$ of $\rho$. Subsequently, we find the roots, and, finally, identify complex frequencies of interest. More precisely, our strategy (the same in both cases) is as follows: First, we calculate the roots of the polynomials $p_{n}$ for $1 \leq n \leq N$ and identify the stable ones. With increasing $n$ the roots migrate on a complex plane and we consider the root as stable, if its location does not change with increasing $n$ to the precision assumed. Sometimes, as we shall see, the roots approach their limit in a quite interesting way. Finally, we accelerate convergence of the series of the root approximants using the well-known Wynn's $\epsilon$ algorithm [26]. In the case in hand, we assume $N=250$ for both the four-term and the three-term recurrences. Since the calculations of the quasinormal modes require knowlegde of all roots of some high-order polynomial one has to use effective and reliable algorithms. Here we have used Jenkins-Traub algorithm and some frequencies has been checked with the aid of the modified Schönhage algorithm ${ }^{1}$.

\footnotetext{
1 The Jenkins-Traub algorithm is default method in Mathematica whereas the modified Schönhage algorithm has been implemented in PARI/GP.
} 


\section{B. The continued fractions method}

As is well known, every three-term recurrence is closely related to some continued fraction. The standard reference for computationally oriented research is the article by Gautschi [27]. Let us recall a few basic facts. The three-term recurrence has generally two independent solutions, and their particular linear combinations, $q_{k}$, with the property that $q_{k} / y_{k} \rightarrow 0$ as $k \rightarrow \infty$, where $y_{k}$ is any solution not proportional to $q_{k}$, form a one-dimensional subspace in the space of all solutions. Elements of this one-dimensional subspace are called minimal (minimal at infinity) and the minimal solution is completely determined by (one) initial value. This is very important because the quasinormal mode corresponds to the minimal solution of a recurrence relations. The convergence condition for the series expansion (which simultaneously is the condition for the quasinormal modes) can be written in the form of the infinite continued fraction

$$
\beta_{0}^{\prime}-\frac{\alpha_{0}^{\prime} \gamma_{1}^{\prime}}{\beta_{1}^{\prime}-\frac{\alpha_{1}^{\prime} \gamma_{2}^{\prime}}{\beta_{2}^{\prime}-\frac{\alpha_{2}^{\prime} \gamma_{3}^{\prime}}{\beta_{3}^{\prime}-\ldots}}}=0
$$

In what follows we shall use the more popular notation, in which the above equation can be written in the form

$$
\beta_{0}-\frac{\alpha_{0} \gamma_{1}}{\beta_{1}-} \frac{\alpha_{1} \gamma_{2}}{\beta_{2}-} \frac{\alpha_{2} \gamma_{3}}{\beta_{3}-} \cdots=0
$$

Inverting Eq. (23) $n$ times, one obtains

$$
\beta_{n}-\frac{\alpha_{n-1} \gamma_{n}}{\beta_{n-1}-} \frac{\alpha_{n-2} \gamma_{n-1}}{\beta_{n-2}-} \cdots-\frac{\alpha_{0} \gamma_{1}}{\beta_{0}}=\frac{\alpha_{n} \gamma_{n+1}}{\beta_{n+1}-} \frac{\alpha_{n+1} \gamma_{n+2}}{\beta_{n+2}-} \frac{\alpha_{n+2} \gamma_{n+3}}{\beta_{n+3}-} \ldots
$$

Both forms are equivalent and may serve as defining equations for calculations of the quasinormal frequancies. Now, our strategy is as follows. First, we generate successive the approximants of the continued fractions up to some $N$ (in our case $n \leq 250$ ) and solve the thus obtained equations. Each approximant is some function of $\omega$. In the next step we identify its stable roots and accelerate convergence of the series of successive approximations to $\omega$ using the $\epsilon$ algorithm. In order to make some independent checks we have used both (24) and (25) and since we are interested in a fundamental modes and their few long-lived overtones, only basic estimations of a tail have been implemented. The more sophisticated calculations would require construction of the asymptotic representation of the remainder of the infinite continued fraction. For the three-term recurrence construction of such asymptotic formula is a five-finger exercise [8, 28], however, as the number of consecutive Gauss eliminations necessary to construct the three-term recurrence grows with the 
dimension $D$ this problem becomes harder and harder [21]. This may lead to the need to modify the computational strategy.

\section{The WKB-Padé approximation}

The WKB-based methods are very popular tools for calculating the frequencies of the quasinormal modes. They are simple, yield reasonable results and depend solely on the derivatives of the potential at its (global) maximum. On the other hand however, they have severe limitations. Indeed, they cannot be used in calculations of the frequencies of higher overtones and may lead to erroneous results for the potentials which are not positive-definite or described by a complicated functions of the radial coordinate with additional maxima and minima [29]. The main idea is simple, and, effectively, it is encapsulated in the formula

$$
\frac{i Q_{0}}{\sqrt{2 Q_{0}^{\prime \prime}}}-\sum_{k=2}^{N} \Lambda_{k}=n+\frac{1}{2},
$$

relating $\omega$, the overtone number $n$ and the functions $\Lambda_{k}$. Each $\Lambda_{k}$ is a combination of the derivatives of $Q(x)=\omega^{2}-V(x)$ calculated at $x=x_{0}$ and its complexity grows fast with the order. Setting all $\Lambda_{k}$ equal to 0 results in the Schutz-Will formula [30, 31, extensively used in the current literature to study large $\ell$ behavior of the quasinormal frequencies. The Schutz-Will formula is the starting point for various generalizations and plays important role in determining the order of magnitude and the general behavior of the modes. Retaining $\Lambda_{2}$ and $\Lambda_{3}$ in 26 gives famous Iyer-Will approximation [13]. Typically, it yields substantially better results that the previous one. The Iyer-Will result has been extended by Konoplya [14 to include the terms up to $N=6$. This method usually gives even more accurate results then the Iyer-Will method, and it seems that it is, in a sense, the optimal one. Due to its simplicity and the quality of the results it gives it is the method of choice in a numerous applications.

The general form of the functions $\Lambda_{k}$ are known for $k \leq 16$ and one can easily incorporate them into the general formula (26). It should be noted however, that since the discussed methods rely on summing up the $\Lambda$ terms they cannot be used to obtain highly accurate complex frequencies. Moreover, increasing the number of $\Lambda$ terms does not improve the quality of the approximation. On the contrary, it can be shown that $\Re(\omega)$ and $|\Im(\omega)|$ rapidly grow with the number of the terms of WKB series summed. 
Our approach, consists of treating the right hand side of the expression

$$
\omega^{2}=V\left(x_{0}\right)-i\left(n+\frac{1}{2}\right) \sqrt{2 Q_{0}^{\prime \prime}} \tilde{\varepsilon}-i \sqrt{2 Q_{0}^{\prime \prime}} \sum_{i=2}^{N} \tilde{\varepsilon}^{j} \Lambda_{j} \equiv V\left(x_{0}\right)+\sum_{i=1}^{N} \tilde{\varepsilon}^{i} \tilde{\Lambda}_{i}
$$

as the power series and instead of summing the terms (which is probably a bad strategy for higherorder calculations given its divergent nature) we construct the Padé approximants [10, 11]. As is well known, the Padé approximants of a truncated power series $\sum a_{k} \tilde{\varepsilon}^{k}$ are defined as the unique rational functions $\mathcal{P}_{N}^{M}(\tilde{\varepsilon})$ of the degree $N$ in the denominator and $M$ in the numerator,

$$
\mathcal{P}_{N}^{M}(\tilde{\varepsilon})=\frac{\sum_{k=0}^{M} A_{k} \tilde{\varepsilon}^{k}}{\sum_{k=0}^{N} B_{k} \tilde{\varepsilon}^{k}}
$$

satisfying simple relation 32$]$

$$
\mathcal{P}_{N}^{M}(\tilde{\varepsilon})-\sum_{k=0}^{M+N} a_{k} \tilde{\varepsilon}^{k}=\mathcal{O}\left(\tilde{\varepsilon}^{M+N+1}\right) .
$$

Without loss of generality one can put $B_{0}=1$. It has been explicitly demonstrated (see Refs. [1012]) that in a number of cases this strategy yields amazingly accurate results. The Padé summation of the WKB terms in Eq. 27] has been proposed in Ref. [10] and subsequently extended in Ref.[11] to which the interested reader is referred for the technical details and a general discussion. Although the functions $\Lambda_{k}$ for $k \geq 17$ are unknown, they can easily be constructed for a given potential with prescribed $\ell$ and $n$ numerically [11, 33, 34]. Finally, observe that instead of the Padé approximants, one can apply the Wynn's algorithm to the partial sums of the series (27).

\section{RESULTS}

\section{A. Massless scalar and gravitational tensor perturbations}

We have used all three methods to calculate the complex frequencies of the quasinormal modes of the massless scalar and gravitational tensor perturbations $(j=0)$ for $\ell=0,1,2$ and their first four overtones (Tables IIIII). Although the calculations have been carried out with a very high precision, the results presented here are rounded to 11 decimal places. Taking $N=250$ both the continued-fraction method and the Hill determinant method with the convergence acceleration give identical result. (Note that the meaning of $N$ in each method is different). In fact, each result displayed in the second column of Tables 파 III has been obtained using four methods: two independent calculations making use of the determinant of the four-diagonal and the tridiagonal matrices and two independent calculations of the continued fractions given by Eqs. (24) and (25), 
respectively. Our general calculational strategy is as follows: as long as possible we try to perform analytical calculations and apply the exact arithmetic. This pays off in the high-precision stage of the numerical calculations.

Our results (when rounded to 4 decimal places) are either in perfect agreement or are very close to the results presented in Ref. [6]. As expected, the WKB-Padé method yields results that are slightly less accurate for the overtones of the fundamental mode, however, it gets progressively better with increase of $\ell$. For example for $\ell \geq 2$ we have a perfect agreement of the methods. The order of the (diagonal) Padé transforms, $\mathcal{P}_{k}^{k}$, depends on the mode and to carry out the calculations in a reasonable time $k$ never exceeds 300 . To simplify calculations we have not attempted to look for smallest optimal order of the Padé transforms that guarantees prescribed accuracy, instead, we have assumed some safe, sufficiently big, value.

Now, let us define deviations of the WKB-Padé results from the results obtained within the framework of the Hill determinant method or the method of continued fractions:

$$
\Delta_{R} \omega=\frac{\Re\left(\omega_{W K B}\right)-\Re\left(\omega_{H D}\right)}{\Re\left(\omega_{H D}\right)} 100 \%
$$

and

$$
\Delta_{I} \omega=\frac{\Im\left(\omega_{W K B}\right)-\Im\left(\omega_{H D}\right)}{\Im\left(\omega_{H D}\right)} 100 \%
$$

A closer examination of Tab. I shows that the deviations of the real part of the frequency never exceed $7 \times 10^{-3} \%$ and $7 \times 10^{-7} \%$ for the real and the imaginary part, respectively. It is an amazing result, which could possibly be made even better by increasing the order of the WKB series and calculating the higher order Padé transforms. Inspection of the Tabs $\Pi$ and $\Pi$ III shows, as expected, that the WKB-Padé method gets progressively better with increase of $\ell$. For the fundamental mode $\ell=1$ and its four first overtones the accuracy of the WKB-Padé based calculations is better than $1.82 \times 10^{-6 \%}$ and $1.16 \times 10^{-7} \%$ for the real and the imaginary part of the frequencies, respectively. For $\ell=2$ we have a perfect agreement of all three methods. 
TABLE I: The frequencies of the quasinormal modes of the scalar and gravitational tensor perturbations $(\ell=0)$ of the five-dimensional Schwarzschild-Tangherlini black hole calculated for $n=0,1,2,3,4$. The Hill determinant method (HD) and the continued-fractions method (CF), both with the convergence acceleration, yield identical results. The WKB-Padé results are slightly less accurate. The frequencies are defined as $\tilde{\omega}=\omega / T_{H}$, where the Hawking temperature $T_{H}=1 / 2 \pi$, and the last column gives the maximal order of the (diagonal) Padé approximants.

\begin{tabular}{|c|c|c|c|}
\hline \hline$n$ & $\tilde{\omega}_{C F} / \tilde{\omega}_{H D}$ & $\tilde{\omega}_{W K B}$ & Padé \\
\hline 0 & $\underline{3.35418783669}-\underline{2.40881848257 i} i$ & $\underline{3.35418783669}-\underline{2.40881848257 i}$ & $(100,100)$ \\
1 & $\underline{2.33646259225}-\underline{8.31019918700 i} i$ & $\underline{2.33646259253-\underline{8.31019918658 i}}$ & $(250,250)$ \\
2 & $\underline{1.88699812446-\underline{14.78624149094 i}}$ & $\underline{1.88699618127-\underline{14.78624157678 i}}$ & $(250,250)$ \\
3 & $\underline{1.69259527010-\underline{21.21981386107 i}}$ & $\underline{1.69260763003-\underline{21.21980227040 i}}$ & $(350,350)$ \\
4 & $\underline{1.58414637600-\underline{27.60684313053 i}}$ & $\underline{1.58403980753-\underline{27.60684306759 i}}$ & $(400,400)$ \\
\hline \hline
\end{tabular}

TABLE II: The frequencies of the quasinormal modes of the scalar and gravitational tensor perturbations $(\ell=1)$ of the five-dimensional Schwarzschild-Tangherlini black hole calculated for $n=0,1,2,3,4$. The Hill determinant method (HD) and the continued-fractions method (CF), both with the convergence acceleration, yield identical results. The WKB-Padé results are slightly less accurate. The frequencies are defined as $\tilde{\omega}=\omega / T_{H}$, where the Hawking temperature $T_{H}=1 / 2 \pi$, and the last column gives the maximal order of the (diagonal) Padé approximants.

\begin{tabular}{|c|c|c|c|}
\hline \hline$n$ & $\tilde{\omega}_{C F} / \tilde{\omega}_{H D}$ & $\tilde{\omega}_{W K B}$ & Padé \\
\hline 0 & $\underline{6.38382253011}-\underline{2.27657411582 i} i$ & $\underline{6.38382253011}-\underline{2.27657411582 i}$ & $(100,100)$ \\
1 & $\underline{5.38079295983}-\underline{7.27345089157 i} i$ & $\underline{5.38079295983}-\underline{7.27345089157 i} i$ & $(150,150)$ \\
2 & $\underline{4.16851774973}-\underline{13.25239223151} i$ & $\underline{4.16851774973}-\underline{13.25239223151} i$ & $(200,200)$ \\
3 & $\underline{3.40134506823}-\underline{19.70845126683 i} i$ & $\underline{3.40134506711-\underline{19.70845126619}}$ & $(300,300)$ \\
4 & $\underline{2.95415287238}-\underline{26.21483703856 i} i$ & $\underline{2.95415281867-\underline{26.21483706910 i}}$ & $(350,350)$ \\
\hline \hline
\end{tabular}

\section{B. The gravitational vector and electromagnetic vector perturbations}

Before we start analysis of the gravitational vector perturbations let us discuss a typical behavior of the Padé approximants of the series given by Eq. (27). A more thorough analysis can be found in Ref. [11. First, observe that for sufficiently large $\ell$ it suffices to retain relatively small number of terms, as the stabilization of the approximants around exact frequency $\omega$ is quite fast. However, for the low-lying fundamental modes and their overtones the stabilization of the results may be slow. Indeed, the approximants may be scattered on the complex plane without any visible pattern or trend, even for a great number of terms retained in the series. However, in many cases, starting 
TABLE III: The frequencies of the quasinormal modes of the scalar and gravitational tensor perturbations $(\ell=2)$ of the five-dimensional Schwarzschild-Tangherlini black hole calculated for $n=0,1,2,3,4$. The Hill determinant method (HD), the continued-fractions method $(\mathrm{CF})$, both with the convergence acceleration, and the WKB-Padé method yield identical results. The frequencies are defined as $\tilde{\omega}=\omega / T_{H}$, where the Hawking temperature $T_{H}=1 / 2 \pi$, and the last column gives the maximal order of the (diagonal) Padé approximants.

\begin{tabular}{|c|c|c|c|}
\hline \hline$n$ & $\tilde{\omega}_{C F} / \tilde{\omega}_{H D}$ & $\tilde{\omega}_{W K B}$ & Padé \\
\hline 0 & $\underline{9.49117521848}-\underline{2.24647282923} i$ & $\underline{9.49117521848}-\underline{2.24647282923 i} i$ & $(100,100)$ \\
1 & $\underline{8.75072206831}-\underline{6.94013514280} i$ & $\underline{8.75072206831}-\underline{6.94013514280} i$ & $(150,150)$ \\
2 & $\underline{7.50107062040}-\underline{12.22543669696 i} i$ & $\underline{7.50107062040}-\underline{12.22543669696 i} i$ & $(200,200)$ \\
3 & $\underline{6.24820058005}-\underline{18.21482743438 i} i$ & $\underline{6.24820058005}-\underline{18.21482743438 i} i$ & $(250,250)$ \\
4 & $\underline{5.31531126304}-\underline{24.59655935942} i$ & $\underline{5.31531126304}-\underline{24.59655935942} i$ & $(300,300)$ \\
\hline \hline
\end{tabular}

with some $N^{\prime}$, the Padé transforms start to stabilize and taking into account additional terms leads to the improvement of the result. Unfortunately, there are cases in which $N^{\prime}$ is either too big to calculate the quasinormal modes in a reasonable time or it does not exist at all. Here we shall adopt a pragmatic point of view: If $N^{\prime}>4 k$, where $k$ is the maximal order of the Padé approximant (in this paper $k=300$ ), then we classify the mode as the mode that cannot be calculated within the WKB-Padé framework. It does not mean that taking larger $k$ would not improve the results. However, it should be emphasized that increasing $k$ may be impractical or even (due to limited computational resources or the very nature of the problem) impossible.

The frequencies of the fundamental quasinormal mode $\ell=2$ and its few lowest overtones are tabulated in Tab. IV] Both the continued fractions method and the Hill determinant method agree to (at least) the quoted accuracy. Unfortunately, the slow convergence of the WKB-Padé approximants for $n=0$, and their apparent lack of stabilization for higher overtones suggest that the WKB-Padé method does not work in this case. Indeed, for $\ell=2, n=0$ mode it yields $\tilde{\omega}=7.15761193-2.11830698 i$ and $\Delta_{R} \omega \approx 0.5 \%$ and $\Delta_{I} \omega \approx 2.7 \%$, which is a poor result.

To examine this discrepancy we introduce the Borel-Le Roy summation [35]. Let $S=\sum_{k} f_{k} \varepsilon^{k}$ be the asymptotic series expansion of some function $f(\varepsilon)$. Now, define a new series by dividing each term by a factor $\Gamma(k+b+1)$

$$
\mathcal{L}^{b}(\varepsilon)=\sum_{k=0}^{\infty} \frac{f_{k}}{\Gamma(k+b+1)} \varepsilon^{k},
$$


where $b(\Re(b)>-1)$ is some adjustable parameter. Since

$$
\Gamma(k+b+1)=\int_{0}^{\infty} d t e^{-t} t^{k+b}
$$

one can use this equation to 'reinsert' the $\Gamma$ factor as follows

$$
f(\varepsilon)=\int_{0}^{\infty} d t e^{-t} t^{b} \mathcal{L}^{b}(\varepsilon t)
$$

Unfortunately, we do not know how to sum the series (32) and if only a finite number of terms is known (and this is the case here) then the integral gives the original series $S$. The essence of the method is to apply the Padé summation to the truncated series 32

$$
S_{B L R}(\varepsilon)=\int_{0}^{\infty} d t e^{-t} t^{b} \mathcal{P}_{N}^{M}(\varepsilon t)
$$

where $\mathcal{P}_{N}^{M}$ is the Padé transform, and to calculate the integral numerically. Putting $b=0$ leads to the Borel summation [32]. The Borel summation of the expansion (27) has been introduced by Hatsuda in Ref. [33] (see also [11, 36]). It should be noted that the integration may be time consuming and numerically unstable.

Now, to minimize the danger of numerical instabilities, we calculate the quasinormal frequency of the $(j=2, l=2, n=0)$ mode using the Borel-Le Roy summation for $b=0,1$ and 2. Quite interestingly, $\omega$ calculated using this technique agrees with the frequency calculated using the WKB-Padé method. Although both WKB-based methods give the same frequency, we believe that its correct value is given by the Hill determinant method and the continued fraction method. On the other hand however, inspection of Tables $\mathrm{V}$ and $\mathrm{VI}$ shows that for $\ell \geq 3$ we have a very good agreement of the results obtained within the frameworks of the all three methods. All this suggests that the WKB-Padé summation is responsible for this discrepancy. It is unclear if retaining more terms in Eq. 27) would improve the WKB result. May be some radical modifications of the method are needed.

Once again, each result presented in the second column of Tables IV VI has been calculated using four methods: two independent calculations making use of the determinant of the fourdiagonal and the tridiagonal matrices and two independent calculations of the continued fractions making use of Eqs. (24) and (25), respectively. We believe that this minimizes the danger of some accidental errors.

For the mode $\ell=3$ and its four first overtones the accuracy of the WKB-Padé based calculations is better than $9.8 \times 10^{-6} \%$ and $3.7 \times 10^{-5} \%$ for the real and the imaginary part of the frequencies, respectively. For $\ell=4$ we have a perfect agreement of all three methods. 
TABLE IV: The frequencies of the quasinormal modes of the gravitational vector perturbations $(\ell=2)$ of the five-dimensional Schwarzschild-Tangherlini black hole calculated for $n=0,1,2,3,4$. The Hill determinant method (HD), the continued-fractions method (CF), both with the convergence acceleration yield identical results. The frequencies are defined as $\tilde{\omega}=\omega / T_{H}$, where the Hawking temperature $T_{H}=1 / 2 \pi$.

\begin{tabular}{|c|c|}
\hline \hline$n$ & $\tilde{\omega}_{C F} / \tilde{\omega}_{H D}$ \\
\hline 0 & $7.12515163375-2.05788530886 i$ \\
1 & $5.95278904708-6.42166605748 i$ \\
2 & $3.41133340871-12.09301936730 i$ \\
3 & $2.73756226817-19.60939205419 i$ \\
4 & $2.51060685222-26.26273928972 i$ \\
\hline
\end{tabular}

TABLE V: The frequencies of the quasinormal modes of the gravitational vector perturbations $(\ell=3)$ of the five-dimensional Schwarzschild-Tangherlini black hole calculated for $n=0,1,2,3,4$. The Hill determinant method (HD) and the continued-fractions method (CF), both with the convergence acceleration, yield identical results. The WKB-Padé results are slightly less accurate. The frequencies are defined as $\tilde{\omega}=\omega / T_{H}$, where the Hawking temperature $T_{H}=1 / 2 \pi$, and the last column gives the maximal order of the (diagonal) Padé approximants.

\begin{tabular}{|c|c|c|c|}
\hline \hline$n$ & $\tilde{\omega}_{C F} / \tilde{\omega}_{H D}$ & $\tilde{\omega}_{W K B}$ & Padé \\
\hline 0 & $\underline{10.84077672458}-\underline{2.09758650296 i} i$ & $\underline{10.84077672458-\underline{2.09758650296 i}}$ & $(200,200)$ \\
1 & $\underline{10.16678579574}-\underline{6.40845617817} i$ & $\underline{10.16678579574}-\underline{6.40845617817} i$ & $(200,200)$ \\
2 & $\underline{8.88194273632-\underline{11.0929034286 i} i}$ & $\underline{8.88194273749-\underline{11.09290342781 i}}$ & $(200,200)$ \\
3 & $\underline{7.21602042842-\underline{16.3979749156 i}}$ & $\underline{7.21602052137-\underline{16.39797479356 i}}$ & $(250,250)$ \\
4 & $\underline{5.527809} 70687-\underline{22.3321576615 i}$ & $\underline{5.527809} 16599-\underline{22.33214931620 i}$ & $(300,300)$ \\
\hline \hline
\end{tabular}

It is of some interest to analyze how the roots of the polynomials $p_{n}$ (see Sec. II A and IIB ) identified as the consecutive approximations of the quasinormal frequencies migrate on the complex plane. For the perturbations considered in this paper it depends on the number of the overtone as follows. Frequency of the fundamental modes and its first overtone $(n=1)$ rapidly approach their limiting values. On the other hand, starting with $n=2$ the approximate frequencies lie on the spiral curve, with the exact value at its center. This behavior is clearly visible in Figs. 1. 3 and 
TABLE VI: The frequencies of the quasinormal modes of the gravitational vector perturbations $(\ell=$ 4) of the five-dimensional Schwarzschild-Tangherlini black hole calculated for $n=0,1,2,3,4$. The Hill determinant method (HD), the continued-fractions method (CF), both with the convergence acceleration, and the WKB-Padé method yield identical results. The frequencies are defined as $\tilde{\omega}=\omega / T_{H}$, where the Hawking temperature $T_{H}=1 / 2 \pi$, and the last column gives the maximal order of the (diagonal) Padé approximants.

\begin{tabular}{|c|c|c|c|}
\hline \hline$n$ & $\tilde{\omega}_{C F} / \tilde{\omega}_{H D}$ & $\omega_{W K B}$ & Padé \\
\hline 0 & $\underline{14.328729987193}-\underline{2.136391879254 i} i$ & $\underline{14.328729987193}-\underline{2.136391879254 i}$ & $(150,150)$ \\
1 & $\underline{13.825163344184}-\underline{6.482259700362 i} i$ & $\underline{13.825163344184}-\underline{6.482259700362 i}$ & $(200,200)$ \\
2 & $\underline{12.850614230172}-\underline{11.057416573784 i} i$ & $\underline{12.850614230172}-\underline{11.057416573784 i}$ & $(200,200)$ \\
3 & $\underline{11.506972273038}-\underline{16.027390914015 i} i$ & $\underline{11.506972273038}-\underline{16.027390914015 i}$ & $(200,200)$ \\
4 & $\underline{9.998966688866}-\underline{21.500865800593 i} i$ & $\underline{9.998966688866}-\underline{21.500865800593 i}$ & $(200,200)$ \\
\hline \hline
\end{tabular}

the red dot at the center represents the result of the application of the $\epsilon$-acceleration to the series of approximate roots. This suggests that the final result is encoded in relatively small number of approximants. Moreover, increasing $n$ while keeping $N$ fixed places the approximants on the complex plane farther from the exact value and to sustain accuracy one has to increase $N$. This remark concerns more the Hill determinant method than the method of continued fraction as in the latter case there is a simple way to estimate the contribution of the remaining part of the continued fractions.

Putting $j=2 / 3$ in Eq. (6) results in the equation considered previously by Crispino, Higuchi, and Matsas (see Eqs. 2.42 and 2.48 in Ref. [37]). Inspection of the Tables VII IX] shows that the results constructed using all three methods are in perfect agreement. The frequencies given by the WKB-Padé method are either exactly the same as the ones calculated using the continued fraction method and the Hill determinant method or slightly less accurate. For example, for $\ell=1$ and $n=4$ (which is the hardest case considered in this paper for the electromagnetic vector perturbations) one has $\Delta_{R} \omega \approx 0.57 \times 10^{-5} \%$ and $\Delta_{I} \omega \approx 0,11 \times 10^{-5} \%$. And this is still amazingly accurate result. The WKB-Padé results can be made even more accurate simply by retaining additional terms in the expansion (27). 


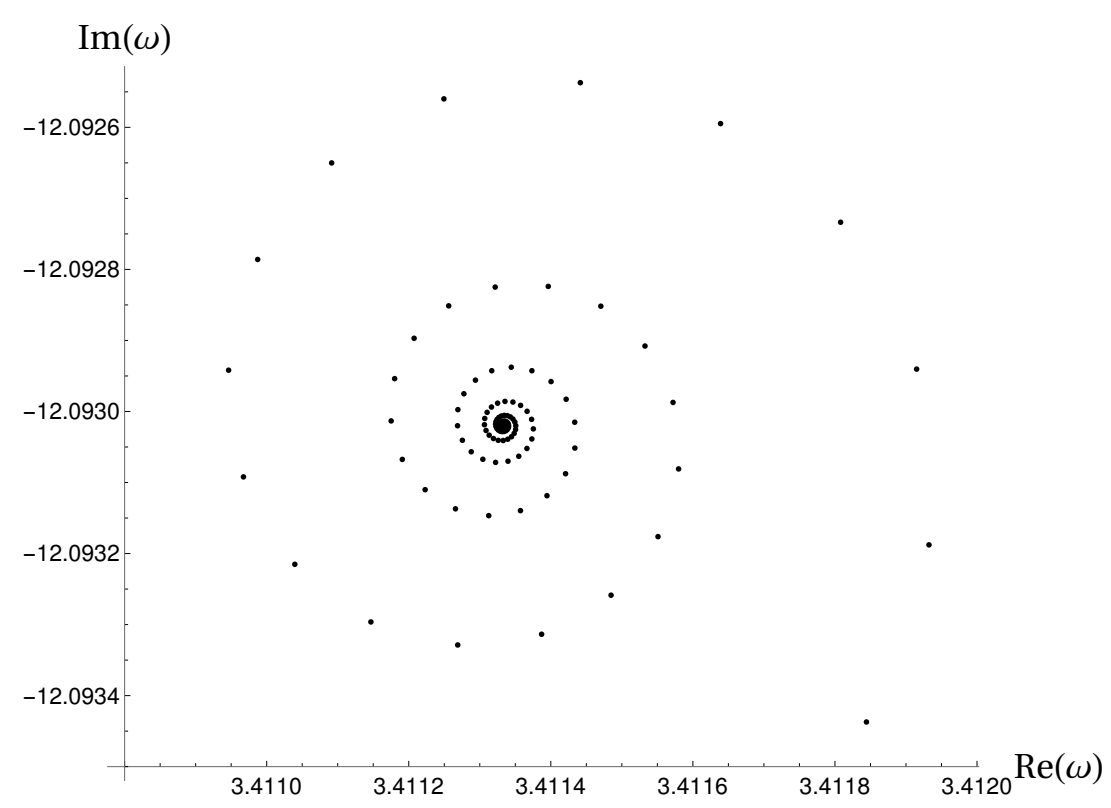

FIG. 1: The gravitational vector perturbations $(j=2)$. Migration of the approximants of the complex frequency of the $\ell=2, n=2$ mode on the complex plane. The red dot represents the limiting value calculated using the Wynn acceleration.

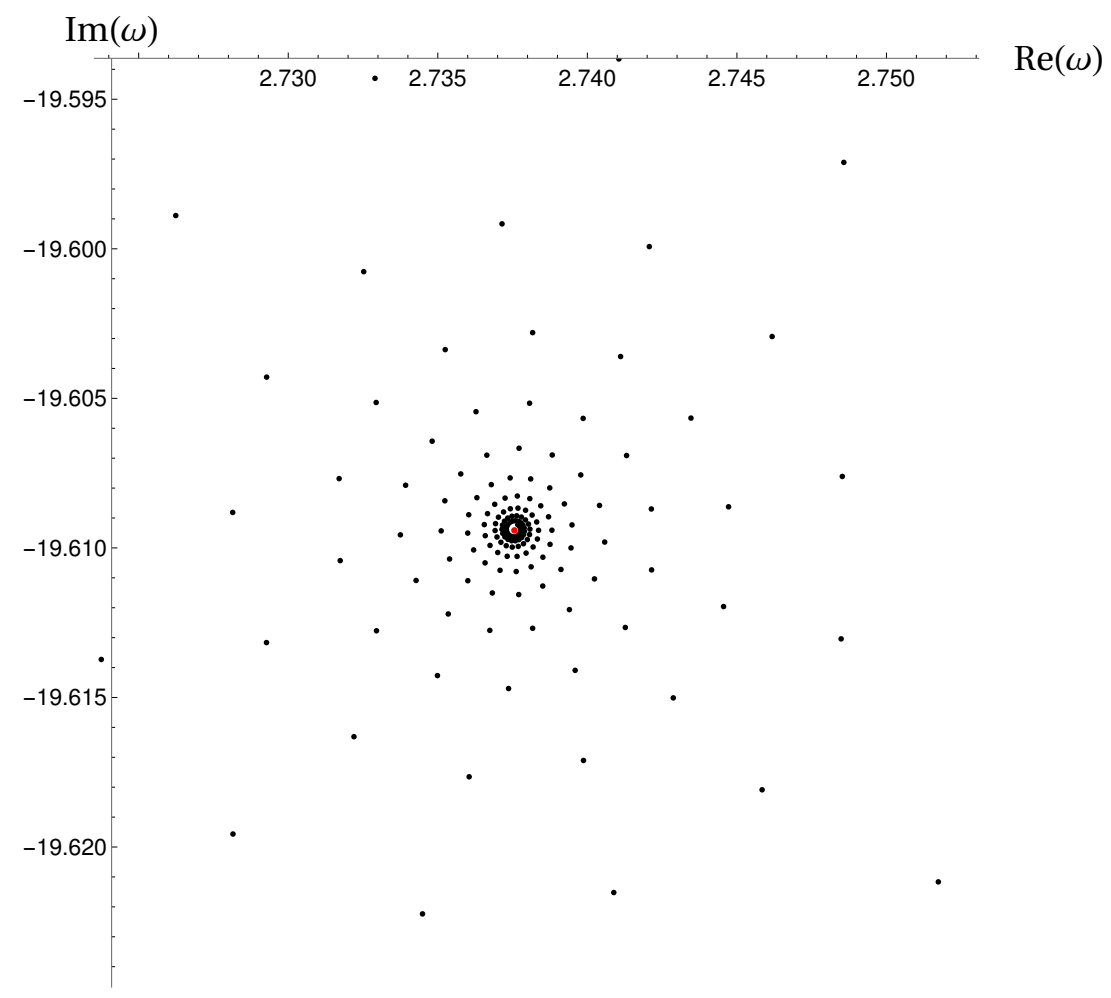

FIG. 2: The gravitational vector perturbations $(j=2)$. Migration of the approximants of the complex frequency of the $\ell=2, n=3$ mode on the complex plane. The red dot represents the limiting value calculated using the Wynn acceleration. 


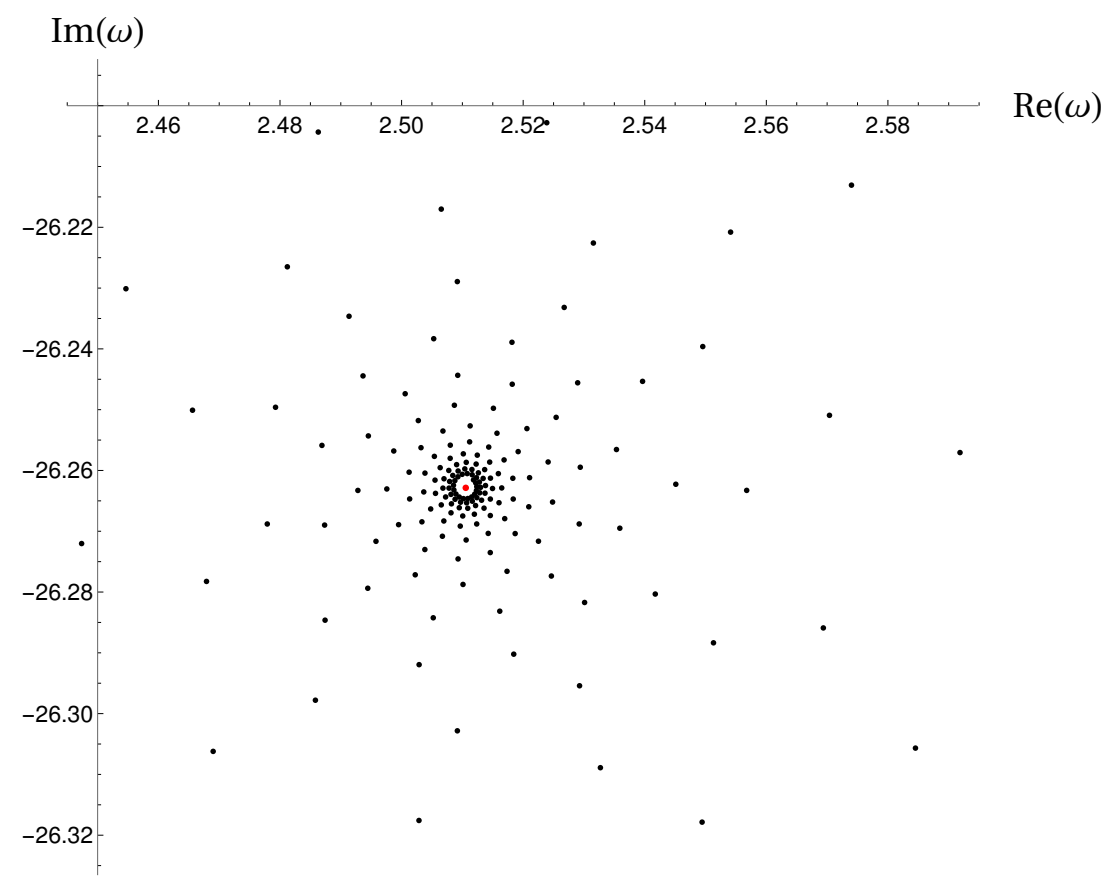

FIG. 3: The gravitational vector perturbations $(j=2)$. Migration of the approximants of the complex frequency of the $\ell=2, n=4$ mode on the complex plane. The red dot represents the limiting value calculated using the Wynn acceleration.

TABLE VII: The frequencies of the quasinormal modes of the electromagnetic vector perturbations $(\ell=$ 1) of the five-dimensional Schwarzschild-Tangherlini black hole calculated for $n=0,1,2,3,4$. The Hill determinant method (HD) and the continued-fractions method (CF), both with the convergence acceleration, yield identical results. The WKB-Padé results are slightly less accurate. The frequencies are defined as $\tilde{\omega}=\omega / T_{H}$, where the Hawking temperature $T_{H}=1 / 2 \pi$, and the last column gives the maximal order of the (diagonal) Padé approximants.

\begin{tabular}{|c|c|c|c|}
\hline \hline$n$ & $\tilde{\omega}_{C F} / \tilde{\omega}_{H D}$ & $\tilde{\omega}_{W K B}$ & Padé \\
\hline 0 & $\underline{5.98616712253}-\underline{2.20376124623 i} i$ & $\underline{5.98616712253}-\underline{2.20376124623 i}$ & $(100,100)$ \\
1 & $\underline{4.93596110359}-\underline{7.06760374979} i$ & $\underline{4.93596110359}-\underline{7.06760374979} i$ & $(100,100)$ \\
2 & $\underline{3.65875874531-\underline{12.9580715859}} i$ & $\underline{3.65875874456-\underline{12.95807158556 i}}$ & $(150,150)$ \\
3 & $\underline{2.836146} 54139-\underline{19.34223930964 i}$ & $\underline{2.83614640894-\underline{19.342239} 20621 i}$ & $(200,200)$ \\
4 & $\underline{2.33220} 463436-\underline{25.78606028998 i} i$ & $\underline{2.33220330869-\underline{25.78606310021 i}}$ & $(250,250)$ \\
\hline \hline
\end{tabular}

\section{DISCUSSION AND FINAL REMARKS}

All perturbations considered so far are described (in a linear regime) by the equation (5), and, consequently, the coefficients $a_{k}$ of the series expansion (10) satisfy the four-term recurrence relation (11). As the continued fraction method requires a three-term recurrence, the relations (11) should 
TABLE VIII: The frequencies of the quasinormal modes of the gravitational vector perturbations $(\ell=$ 2 ) of the five-dimensional Schwarzschild-Tangherlini black hole calculated for $n=0,1,2,3,4$. The Hill determinant method (HD) and the continued-fractions method (CF), both with the convergence acceleration, yield identical results. The WKB-Padé results are almost as accurate as the previous two. The frequencies are defined as $\tilde{\omega}=\omega / T_{H}$, where the Hawking temperature $T_{H}=1 / 2 \pi$, and the last column gives the maximal order of the (diagonal) Padé approximants.

\begin{tabular}{|c|c|c|c|}
\hline \hline$n$ & $\tilde{\omega}_{C F} / \tilde{\omega}_{H D}$ & $\tilde{\omega}_{W K B}$ & Padé \\
\hline 0 & $\underline{9.2271133280308}-\underline{2.2143549304187 i} i$ & $\underline{9.2271133280308}-\underline{2.2143549304187 i} i$ & $(100,100)$ \\
1 & $\underline{8.4728284656951}-\underline{6.8458616593904 i} i$ & $\underline{8.4728284656951}-\underline{6.8458616593904 i} i$ & $(100,100)$ \\
2 & $\underline{7.1966403739904}-\underline{12.0804073774333 i} i$ & $\underline{7.1966403739904}-\underline{12.0804073774333 i} i$ & $(150,150)$ \\
3 & $\underline{5.9189890960475}-\underline{18.0342846521014 i} i$ & $\underline{5.9189890960475}-\underline{18.0342846521015 i}$ & $(150,150)$ \\
4 & $\underline{4.9682509345537}-\underline{24.3832179197589} i$ & $\underline{4.9682509345535}-\underline{24.3832179197585 i}$ & $(250,250)$ \\
\hline \hline
\end{tabular}

TABLE IX: The frequencies of the quasinormal modes of the electromagnetic vector perturbations $(\ell=$ 3) of the five-dimensional Schwarzschild-Tangherlini black hole calculated for $n=0,1,2,3,4$. The Hill determinant method (HD), the continued-fractions method $(\mathrm{CF})$, both with the convergence acceleration, and the WKB-Padé method yield identical results. The frequencies are defined as $\tilde{\omega}=\omega / T_{H}$, where the Hawking temperature $T_{H}=1 / 2 \pi$, and the last column gives the maximal order of the (diagonal) Padé approximants.

\begin{tabular}{|c|c|c|c|}
\hline \hline$n$ & $\tilde{\omega}_{C F} / \tilde{\omega}_{H D}$ & $\tilde{\omega}_{W K B}$ & Padé \\
\hline 0 & $\underline{12.4184186551775}-\underline{2.2177094497067 i} i$ & $\underline{12.4184186551775}-\underline{2.2177094497067 i}$ & $(100,100)$ \\
1 & $\underline{11.8412014398195}-\underline{6.7660501645214} i$ & $\underline{11.8412014398195}-\underline{6.7660501645214 i} i$ & $(150,150)$ \\
2 & $\underline{10.7694063181563}-\underline{11.6625811156602} i$ & $\underline{10.7694063181563}-\underline{11.6625811156602} i$ & $(150,150)$ \\
3 & $\underline{9.4315982617932}-\underline{17.1019392490840} i$ & $\underline{9.4315982617932}-\underline{17.1019392490840} i$ & $(200,200)$ \\
4 & $\underline{8.1520905535350}-\underline{23.0769667314609} i$ & $\underline{8.1520905535350}-\underline{23.0769667314609} i$ & $(200,200)$ \\
\hline \hline
\end{tabular}

be transformed to the required form by the Gauss elimination. Since we are interested in the lowlying fundamental modes and their overtones satisfying $(n \leq 4)$ we employed the series acceleration technique. On the other hand, in the Hill-determinant method one can use both the four-term or the three-term recurrence. The determinant of $(n+1 \times n+1)$ matrix constructed form the infinite sparse band matrix of width 4 can be calculated efficiently with the aid our formula (16). Similarly, one can calculate the determinant of the tridiagonal matrix. A typical migration of the roots that approximate quasinormal frequency on the complex plane is shown in Figs. 1,3. For the low-lying overtones, the roots approach their limiting value quite fast, whereas the general trend for higher overtones suggests that the convergence becomes slower and slower. To secure great accuracy of 
the quasinormal frequencies for the higher overtone numbers, the dimension of the matrices should be unreasonably big and to accelerate convergence we have used, once again, the Wynn's $\epsilon$-method. On the other hand, the gravitational scalar perturbations described by the potential (7) lead to the eight-term recurrence. Since (7) does not belong to the class of potentials considered in this paper, here we report only on our preliminary results obtained within the framework of the WKB-Padé approximation. The tables of the quasinormal frequencies (to 4 decimal places) calculated using the method of continued fractions are given in Ref. [7]. Our results for $\ell=2$ (when transformed to the normalization adopted in [7]) are $0.94774-0.25609 i, 0.85123-0.82116 i, 0.67274-1.54307 i$ and $0.50889-2.43311 i$ for $n=0,1,2$, and 3, respectively, and when rounded to four decimal places they are exactly the same as those presented in Ref. [7]. This shows the power of the WKB-Padé method and simultaneously confirms correctness of the results obtained by Cardoso, Lemos and Yoshida. It should be noted, that because of a complicated form of the gravitational scalar potential, the calculations are much more involved.

Let us return to the Hill determinant method applied to perturbations leading to $k$-term recurrences $(k>3)$. As has been explained earlier, one can use either the Gauss elimination to obtain the tridiagonal matrix or work with the original recurrence. To calculate determinants in the latter case efficiently, one should generalize Eq. (16). In real calculations the computational complexity of each method should be estimated, i.e, the following question should be asked. What is more time-consuming: Calculations of determinants of banded matrices of width $k$ with relatively simple matrix elements, or performing $(k-3)$ consecutive Gauss eliminations and calculating determinants of tridiagonal matrices with quite complicated elements?

It should be emphasized that the perfect agreement between the results obtained using the Hill determinant method and the method of continued fractions, although expected, is quite impressive and the role of the convergence acceleration should not be underestimated in this regard. On the other hand, the performance of the WKB-Padé method (within domain of its applicability) is really amazing. In most cases it is better than any competing WKB-based technique. Of course, such a comparison is somewhat unfair given the much greater complexity of our method.

The techniques presented in this paper can easily be adapted to other dimensions. However, there are a few bottlenecks that may pose a challenge to the calculations of the quasinormal modes. For the method of continued fractions and the Hill determinant method it would be a complexity of the equations which have to be solved. At any order one has to identify all solutions and this may be a time-consuming process. On the other hand, the real problem of the WKB-Padé method is the necessity to calculate the consecutive derivatives of the potential at its maximum. Since 
the calculations are carried out analytically it places severe demand on the computer resources, especially for more complex potentials. Moreover, it turns out that the time spent on construction of the Padé transforms is only a small fraction of the total time of computations and for a given $N$, the calculation time of the WKB series is practically insensitive to the type of the black hole perturbation.

Finally, let us briefly discuss the strength and limitations of each method employed in this paper. Of course, their great positive aspect is the ability to construct the highly accurate values of $\omega$. However, they lose accuracy with increasing the overtone number, $n$, even though we use the convergence acceleration algorithms. For the continued fraction method and the method of the Hill determinant the deterioration of the results is slow, and to secure assumed accuracy it is sufficient to increase the number of terms retained in the expansion (10). For highly damped modes this may be impractical or insufficient. In such a case it would be reasonable to construct the asymptotic approximation of the remainder of the continued fraction and to modify the root searching algorithm. On the other hand, the WKB-Padé method has limited applicability for overtones of the low lying modes, which is reflected in slow stabilization (if any) of the series of approximants. This can be deduced form the trend clearly visible in the tables. It is not clear if increasing the number of terms in (27) would cure the problem. Such calculations are both demanding and time consuming and we intend to return to this group of problems elsewhere.

[1] K. D. Kokkotas and B. G. Schmidt, Living Rev. Rel. 2, 2 (1999).

[2] E. Berti, V. Cardoso, and A. O. Starinets, Class. Quant. Grav. 26, 163001 (2009).

[3] R. A. Konoplya and A. Zhidenko, Rev. Mod. Phys. 83, 793 (2011).

[4] H.-P. Nollert, Class. Quant. Grav. 16, R159 (1999).

[5] P. Pani, Int. J. Mod. Phys. A 28, 1340018 (2013), 1305.6759.

[6] V. Cardoso, J. P. S. Lemos, and S. Yoshida, Phys. Rev. D 69, 044004 (2004).

[7] V. Cardoso, J. P. S. Lemos, and S. Yoshida, JHEP 12, 041 (2003).

[8] E. W. Leaver, Proc. Roy. Soc. Lond. A402, 285 (1985).

[9] B. Majumdar and N. Panchapakesan, Phys. Rev. D 40, 2568 (1989).

[10] J. Matyjasek and M. Opala, Phys. Rev. D 96, 024011 (2017).

[11] J. Matyjasek and M. Telecka, Phys. Rev. D 100, 124006 (2019).

[12] J. Matyjasek, Phys. Rev. D 102, 124046 (2020), 2009.10793.

[13] S. Iyer and C. M. Will, Phys. Rev. D 35, 3621 (1987).

[14] R. A. Konoplya, Physical Review D 68, 024018 (2003). 
[15] P. P. Fiziev (2019), arXiv:1912.13432.

[16] D. Birmingham, Phys. Lett. B 569, 199 (2003).

[17] R. A. Konoplya, Phys. Rev. D 68, 124017 (2003).

[18] A. Rostworowski, Acta Phys. Polon. B38, 81 (2007).

[19] E. Berti, M. Cavaglia, and L. Gualtieri, Phys. Rev. D 69, 124011 (2004).

[20] D. Yoshida and J. Soda, Phys. Rev. D 99, 044054 (2019).

[21] E. W. Leaver, Phys. Rev. D 41, 2986 (1990).

[22] V. Cardoso and J. P. S. Lemos, Phys. Rev. D 66, 064006 (2002).

[23] A. Ishibashi and H. Kodama, Prog. Theor. Phys. 110, 901 (2003).

[24] H. Kodama and A. Ishibashi, Prog. Theor. Phys. 111, 29 (2004).

[25] G. Gibbons and S. A. Hartnoll, Phys. Rev. D 66, 064024 (2002).

[26] P. Wynn, Mathematical Tables and Other Aids to Computation pp. 91-96 (1956).

[27] W. Gautschi, SIAM review 9, 24 (1967).

[28] H.-P. Nollert, Phys. Rev. D 47, 5253 (1993).

[29] G. Khanna and R. H. Price, Phys. Rev. D 95, 081501 (2017).

[30] B. F. Schutz and C. M. Will, Astrophys. J. 291, L33 (1985).

[31] B. Mashhoon, in Third Marcel Grossmann Meeting on General Relativity (North-Holland, 1983), p. 599 .

[32] C. M. Bender and S. A. Orszag, Advanced mathematical methods for scientists and engineers (McGrawHill, New York, 1978).

[33] Y. Hatsuda, Phys. Rev. D 101, 024008 (2020).

[34] T. Sulejmanpasic and M. Ünsal, Comput. Phys. Commun. 228, 273 (2018).

[35] É. Le Roy, Annales de la Faculté des sciences de Toulouse: Mathématiques 2, 317 (1900).

[36] D. S. Eniceicu and M. Reece, Phys. Rev. D 102, 044015 (2020).

[37] L. C. B. Crispino, A. Higuchi, and G. E. A. Matsas, Phys. Rev. D 63, 124008 (2001), [Erratum: Phys. Rev. D 80, 029906 (2009)]. 